\title{
Factores de salud percibida y de calidad de vida al inicio de la educación universitaria en Colombia
}

\author{
Fabián Bravo; Maria Fernanda Parra; Luis Manuel Silva
}

Cómo citar este artículo:

Bravo, F., Parra, M. F., \& Silva, L. M. (2021). Factores de salud percibida y de calidad de vida al inicio de la educación universitaria en Colombia. Acta Colombiana de Psicología, 24(2), 59-68. https://www.doi.org/10.14718/ACP.2021.24.2.6

Recibido, marzo 11/2020; Concepto de evaluación, diciembre 11/2020; Aceptado, abril 28/2021

\author{
Fabián Bravo ${ }^{1}$ \\ ORCID: https://orcid.org/0000-0002-1455-1554 \\ Pontificia Universidad Javeriana, Cali, Colombia. \\ Maria Fernanda Parra \\ ORCID: https://orcid.org/0000-0002-3417-1137 \\ Pontificia Universidad Javeriana, Cali, Colombia. \\ Luis Manuel Silva \\ ORCID: https://orcid.org/0000-0001-7051-0186 \\ Pontificia Universidad Javeriana, Bogotá, Colombia.
}

\begin{abstract}
Resumen
El objetivo del presente estudio fue identificar factores de salud percibida y de calidad de vida en estudiantes de primer semestre de una universidad privada en Colombia. Para esto, se contó con una muestra por conveniencia de 636 estudiantes $(M=18$ años, $D E=1.38)$ que participó en la primera medición de una investigación longitudinal denominada VIDA2020. En particular, se realizó un diseño de investigación observacional transversal, enmarcado en una investigación de cohorte. Del protocolo de evaluación del proyecto, este reporte incluye un cuestionario con datos sociodemográficos y antecedentes médicos, las subescalas de depresión y estrés del DASs-21, un ítem del sF36v-2 y el WHOQOL-BREF. En general, como resultado se encontró que el $91 \%$ de los estudiantes valora su salud global como buena o excelente; que dicha valoración es significativamente menor en quienes reportan antecedentes de enfermedad (80.5\%) y síntomas de depresión $(86.1 \%)$ y estrés (83.1\%); que las mujeres, los migrantes y quienes reportan antecedentes de enfermedad puntúan menor calidad de vida física, psicológica y ambiental; y que el estrés y la depresión predicen menores puntajes de calidad de vida física y psicológica, con correlaciones más fuertes en comparación con las variables demográficas y médicas. Estos hallazgos permiten identificar y priorizar las necesidades especiales en salud de los nuevos estudiantes.

Palabras clave: salud percibida, calidad de vida, universitarios, primer año, Colombia.
\end{abstract}

1 Calle 18 No. 118-250, Departamento de Ciencias Sociales, Facultad de Humanidades y Ciencias Sociales, Pontificia Universidad Javeriana, Cali, Colombia. Tel.: (+57-2) 3218200. fbravo@javerianacali.edu.co

Este trabajo recibió financiación de la Pontificia Universidad Javeriana, con los proyectos 8492 de la sede central y 4434 de la seccional Cali. 


\title{
Perceived health and quality of life factors at the beginning of university education in Colombia.
}

\begin{abstract}
The aim of this study was to identify factors of perceived health and quality of life in first semester students of a private university in Colombia. For this purpose, a convenience sample of 636 students $(M=18$ years, $S D .=1.38)$ participated in this first phase of the longitudinal study called VIDA2020. An observational cross-sectional research design framed within a cohort study was used. From the assessment protocol of the project, this report includes a questionnaire with sociodemographic data and medical history, the DASS-21 depression and stress subscales, one item of the SF36V-2, and the WHOQOL-BREF. Results showed that most students (91\%) rated their overall health as good or excellent, but this rating was significantly lower in those reporting a history of illness in their medical background (80.5\%) and symptoms of depression $(86.1 \%)$ and stress $(83.1 \%)$. Women, migrants and those reporting a history of illness scored lower on physical, psychological and environmental dimensions of quality of life. Additionally, stress and depression predict lower physical and psychological quality of life scores, with stronger correlations compared to sociodemographic and medical variables. These findings allow the identification and prioritization of special health needs of new students.

Keywords: perceived health, quality of life, university students, first year, Colombia.
\end{abstract}

\section{Introducción}

Como se encuentra en diversas investigaciones, los jóvenes inician la educación universitaria con diferencias relevantes en sus antecedentes médicos y estado de salud (Chau \& Saravia, 2014; Lolokote et al., 2017), de manera que para promover la salud dentro del campus es fundamental identificar sus necesidades especiales, incluyendo la prevalencia de enfermedades crónicas, limitaciones funcionales, quejas en salud y hábitos de autocuidado (Arroyo et al., 2006; Chu et al., 2015; Intra et al., 2011). Incluso, se debe reconocer su salud mental inicial (Bayram $\&$ Bilgel, 2008), los patrones de comportamiento saludable y no saludable (Barnett et al., 2014), y la valoración que hacen de su propia salud (Chau \& Saravia, 2014; Lolokote et al., 2017; Mikolajczyk et al., 2008) y de su calidad de vida (Chu et al., 2015; El Ansari et al., 2011).

Cumplir lo anterior es imperativo en países como Colombia, donde la composición social y procedencia regional de los universitarios se ha hecho cada vez más heterogénea tanto en instituciones de educación superior públicas como privadas. Esta mayor diversidad social está relacionada con un incremento en la cobertura de la educación superior, que ha pasado del $28.4 \%$ en 2005 al $47 \%$ en 2015; apalancada, entre otras razones, por una mayor oferta de créditos y becas para aspirantes de menores recursos, como es el caso del programa Ser Pilo Paga (Resolución 175 de 2018) del Ministerio de Educación Nacional de Colombia (MEN, 2015), actualmente denominado Generación E.

Adicional a esta justificación contextual, los universitarios, como jóvenes que son, constituyen a nivel global un segmento de la población de elevada prioridad (Sawyer et al., 2012), debido a sus menores beneficios en la denominada transición epidemiológica en comparación con niños y adultos, y también por la inadecuada o insuficiente atención que han recibido sus necesidades en salud (Resnick et al., 2012). En algunos países, incluso, su mortalidad es mayor en comparación con las edades precedentes, y las causas son en su mayoría prevenibles. Entre estas figuran el suicidio, los accidentes de tránsito, la violencia, el SIDA y la muerte materna por embarazo adolescente (Patton et al., 2009).

A la elevada mortalidad se suma una importante prevalencia de comportamientos de riesgo y de alteraciones de la salud mental de los jóvenes a nivel mundial (Patton et al., 2014). En este particular, en una revisión de estudios publicados desde 1995 se identificó tasas de desórdenes mentales y abuso de sustancias que van desde el 8 hasta el $57 \%$ (Patel et al., 2007). Aunque las cifras cambian considerablemente de un país a otro, de la misma revisión se deriva que mundialmente al menos uno de cada cuatro jóvenes presenta como mínimo un desorden mental.

Frente a este panorama, y con el objetivo de mejorar la salud de los jóvenes, se ha resaltado la importancia de reconocerlos en el marco del ciclo vital, de los contextos en los que se desarrollan — familia, escuela, universidad y comunidad-y de los periodos de transición (Patton et al., 2016); donde se destaca la universidad como un entorno y periodo clave (Barnett et al., 2014), ideal para el estudio y promoción de la salud presente y futura (Bayram \& Bilgel, 2008; Conley, Durlak et al., 2013; Conley, Travers et al., 2013; Hee-Kyung et al., 2017).

Consecuente con lo anterior, en la literatura especializada muchos estudios incluyen muestras de universitarios, lo que los convierte en una población bien representada. 
Sin embargo, la salud en el primer semestre no ha recibido suficiente atención, por lo cual se pierde la posibilidad de estudiar el ingreso a la universidad como una etapa caracterizada por intensos cambios en la dimensión personal y familiar (Azmitia et al., 2013; Conley, Durlak et al., 2013), y social y educativa (Bayram \& Bilgel, 2008; Conley, Durlak et al., 2013; Conley, Travers et al., 2013; Dvoráková et al., 2017; El Ansari et al., 2011).

Fuera del contexto latinoamericano se han realizado valiosos estudios de transición a la universidad con enfoque en la salud de los estudiantes (Ari \& Shulman, 2012; Fielder et al., 2014; Han et al., 2008; Patrick \& Lee, 2010; Racette et al., 2014; Wengreen \& Moncur, 2009); no obstante, el foco de dichos estudios se dirige principalmente a los factores y comportamientos de riesgo para la salud (Ari \& Shulman, 2012; Fielder et al., 2014; Han et al., 2008; Patrick \& Lee, 2010; Racette et al., 2014; Wengreen \& Moncur, 2009) y al compromiso de la salud mental (Ari \& Shulman, 2012; Fielder et al., 2014), con una menor consideración de la salud desde la perspectiva del propio joven.

Entre las investigaciones disponibles de enfoque positivo se destaca el estudio de la calidad de vida de los universitarios (El Ansari et al., 2011), el de la percepción global de su salud de un solo ítem (Chau \& Vilela, 2017; Chu et al., 2015; El Ansari et al., 2011; Mikolajczy et al., 2008), el apoyo social percibido (Chu et al., 2015; El Ansari et al., 2011), el ajuste psicosocial a la universidad (Chau \& Saravia, 2014; Conley, Traver, 2013; Figuera et al., 2003; Londoño, 2009), y el optimismo (Londoño, 2009), siendo los dos primeros los que recientemente han recibido mayor atención. Entre los hallazgos más consistentes, destaca que entre el $80 \%$ y $90 \%$ de los universitarios de diferentes países percibe su salud como buena o excelente (Chu et al., 2015; El Ansari et al., 2011; Intra et al., 2011; Mikolajczy et al., 2008), y que dicha percepción es peor en las mujeres (Chau \& Vilela, 2017; El Ansari et al., 2011; Mikolajczy et al., 2008). Sin embargo, en este último particular se ha concluido que dicha diferencia se debe exclusivamente a que los hombres califican mejor su salud psicológica que las mujeres (Mikolajczy et al., 2008); diferencia específica también identificada con respecto a la calidad de vida en la dimensión psicológica (El Ansari et al., 2011).

De manera general, otros factores asociados a la percepción de salud incluyen la salud física y psicológica, así como las características sociodemográficas de los estudiantes (Chau \& Vilela, 2017; Chu et al., 2015; Lolokote et al., 2017; Mikolajczy et al., 2008). De hecho, se han encontrado relaciones significativas con el nivel educativo y estatus financiero del estudiante (Lolokote et al., 2017), el país de residencia (Mikolajczy et al., 2008), la convivencia con los padres (Chau \& Vilela, 2017; Mikolajczy et al., 2008) y el nivel educativo de la madre (Mikolajczy et al., 2008); no obstante, estos resultados no son generalizables al inicio de los estudios universitarios en el contexto latinoamericano, ni a Colombia en particular.

Considerando lo anterior, la pregunta que subyace a este trabajo es "¿qué variables del estudiante de primer semestre se relacionan con la percepción de su salud y con su calidad de vida en Colombia?". Las variables especificas consideradas son el sexo, el nivel socioeconómico, la financiación y migración por estudios, la enfermedad crónica, el índice de masa corporal, la depresión y el estrés. Lo anterior debido a que se maneja como hipótesis una significativa mejor salud percibida y mejor calidad de vida en estudiantes hombres que reciben apoyo financiero de sus padres, sin diagnóstico de enfermedad crónica y sin sintomatología relevante de depresión y estrés.

De acuerdo con esto, los objetivos del presente trabajo fueron: (a) describir los antecedentes médicos y la sintomatología de depresión y estrés, diferenciando las medidas por sexo; (b) relacionar la percepción de la salud y la calidad de vida con variables sociodemográficas, médicas y de salud emocional — depresión y estrés - ; y, finalmente, (c) identificar predictores independientes de calidad de vida física y psicológica.

\section{Método}

Tipo de estudio y diseño

Este estudio es resultado de la primera fase (primera medición) de un proyecto longitudinal denominado VIDA2020, diseñado en la Pontificia Universidad Javeriana en Colombia con el propósito de estudiar el curso de la salud durante los tres primeros años de educación universitaria. Por incluir una sola evaluación de la cohorte, se caracteriza como una investigación observacional transversal, enmarcada en un estudio de cohorte (Ato et al., 2013).

\section{Participantes}

Se utilizó una muestra por conveniencia que incluyó 636 estudiantes de primer semestre (423 de la ciudad de Cali y 213 de Bogotá), con edad promedio de 18 años $(D E=1.38)$ y mayor representación de mujeres (57.2\%). Al momento de la evaluación, el $78.8 \%$ reportó vivir con sus padres, el $25.9 \%$ informó que se financiaba con créditos o becas, el $22 \%$ cambió de ciudad para entrar a la universidad, y solo el $3.9 \%$, además de estudiar, trabajaba. El nivel socioeconómico medio fue el de mayor representación, con $49.1 \%$, seguido del alto, con $41.5 \%$, y el bajo, con solo el $7.2 \%$. 
62

\section{Instrumentos}

El protocolo inicial del proyecto VIDA2020 incluyó un cuestionario de datos sociodemográficos y antecedentes médicos; tres escalas de salud —el Depression, Anxiety and Stress Scales (DASs-21; Lovind \& Lovind, 1995), el Short Form 36-V2 (sF36 v.2; Maruish, 2011) y el World Health Organization Quality of Life, versión breve (WHOQOL-BREF; wHO, 1998) - ; y una encuesta de estilos de vida diseñada por el equipo investigador a partir de instrumentos disponibles. A continuación se describen los instrumentos usados exclusivamente para este trabajo.

\section{Datos personales y antecedentes médicos}

Para obtener información sociodemográfica y de antecedentes en salud, se adaptó el cuestionario usado por la Universidad Javeriana para caracterizar a sus nuevos estudiantes. Este incluye, entre otras variables, el lugar de residencia, con quién vive el estudiante y la fuente de financiación de los estudios. Por otra parte, como antecedentes médicos, se preguntó la estatura y el peso para calcular el índice de masa corporal (IMC), la presencia de enfermedad física o mental diagnosticada por especialista $-\mathrm{y}$ también de discapacidad o limitación funcional—, y la utilización de servicios médicos.

\section{Depresión y estrés}

Como indicadores de salud emocional se tomaron las subescalas de depresión y estrés del DASS-21 (Lovibond \& Lovibond, 1995). Cada una cuenta con 7 ítems que indagan respecto a síntomas como desesperanza, baja autoestima y poco afecto positivo (subescala de depresión), y otros como dificultad para relajarse, sobreactivación e intolerancia (subescala de estrés). El DAss-21 es un instrumento ampliamente utilizado para el tamizaje de depresión, ansiedad y estrés; cuenta con propiedades psicométricas adecuadas (alfa de Cronbach $=.92$ para la escala total); y su estructura factorial se ha confirmado en Colombia (Ruiz et al., 2017). Específicamente, la escala de respuesta es ordinal de cuatro categorías (0-3), que van desde "no me aplicó" el síntoma descrito hasta "me aplicó mucho o la mayor parte del tiempo"; y el manual de prueba ofrece puntos de corte para obtener cinco niveles de severidad, desde normal (depresión $<10$ y estrés $<15$ ) hasta extremadamente severo (Lovibond \& Lovibond, 1995).

\section{Salud percibida}

Como indicador de salud percibida se tomó el primer ítem del SF36 v.2 (Maruish, 2011), que indaga por la percepción general de la salud, "en general, diría usted que su salud es...", con cinco opciones de respuesta, que van de "excelente" a "mala". Las respuestas pueden ser recodificadas en dos categorías de percepción de la salud, (a) buena o excelente y (b) regular o mala, para favorecer la comparabilidad con estudios disponibles.

\section{Calidad de vida}

Para evaluar la calidad de vida se administró el WHOQOLBREF (WHO, 1994), que es la versión resumida WHOQOL de 100 ítems diseñado por la Organización Mundial de la Salud (wHO, 1994). Según su fundamentación, este instrumento es sensible al contexto y al sistema de valores de los evaluados, así como a sus metas, expectativas y preocupaciones propias (The World Health Organization Quality of Life Group, 1994). Específicamente, el instrumento está compuesto por cuatro dimensiones de la calidad de vida: (a) calidad de vida fisica, p. ej., dolor, fatiga, energía, movilidad, capacidad laboral, etc.; (b) calidad de vida psicológica, p. ej., emociones, memoria, capacidad de concentración, imagen corporal, espiritualidad, etc.; (c) calidad de vida social, p. ej., relaciones interpersonales, percepción de apoyo, sexualidad, etc.; y (d) calidad de vida ambiental, p. ej., seguridad física y libertad, accesibilidad a servicios, oportunidades de ocio, transporte, etc. Los estudios de validación del WHOQOL-BREF presentan propiedades psicométricas apropiadas —alfa para las dimensiones entre .66 y .84, correlaciones con la versión de 100 ítems entre .89 y .95 , y confirmación de la estructura factorial-, y se dispone de una versión en español proporcionada por la OMS (WHO, 1998), que fue la que se utilizó en el presente trabajo. Para los análisis, las puntuaciones directas se transformaron linealmente a una escala de 0 a 100, correspondientes a la peor y mejor calidad de vida posible en las respectivas dimensiones.

\section{Procedimiento}

Para participar en el proyecto VIDA2020, los estudiantes debían estar matriculados en primer semestre al momento de inicio del estudio y diligenciar los instrumentos completos — con no más del $5 \%$ de información perdida- L La recolección de la información se realizó con cuestionarios físicos - papel y lápiz- diligenciados en las aulas de clase con las respectivas autorizaciones de la universidad. En unos pocos casos $(n=25)$, el cuestionario se diligenció virtualmente, con una versión digital de la batería de instrumentos.

\section{Consideraciones éticas}

El proyecto fue aprobado por el comité de ética de la Facultad de Humanidades y Ciencias Sociales de la institución que acogió el estudio. Todos los participantes firmaron los consentimientos informados, y, para el caso de 
menores de edad, los padres o tutores legales firmaron el consentimiento informado y los participantes un asentimiento informado. Los documentos de consentimiento incluyeron las garantías de confidencialidad, participación voluntaria $\mathrm{y}$ derecho al retiro en cualquier momento. Adicionalmente, se incluía que el seguimiento a la salud y al desempeño académico se realizaría durante tres años consecutivos, razón por la cual serían nuevamente contactados.

\section{Análisis de datos}

El resumen de las variables cualitativas se realizó con frecuencias y porcentajes, y el de las cuantitativas con promedios y desviaciones estándar. Por otra parte, los análisis bivariados de los antecedentes médicos, sintomatología de depresión y estrés y percepción general de la salud fueron realizados con análisis de Chi-cuadrado; y para las comparaciones entre grupos en las puntuaciones de calidad se implementó la opción paramétrica, aunque los datos no se ajustaron a la distribución normal según la prueba de Kolmogorov-Smirnov. Lo anterior atendiendo las condiciones de robustez del estadístico $t$ student referidas al poco efecto del alejamiento de la normal cuando se dispone de muestras grandes (Gravetter \& Wallnau, 2013; Lumley et al., 2002; McGrath, 2012).

Finalmente, se aplicaron análisis de regresión lineal múltiple para identificar predictores independientes de la calidad de vida exclusivamente en los dominios de mayor pertinencia para esta población - física y psicológicaPara establecer la validez de los modelos, se evaluó la distribución de los errores y la ausencia de autocorrelación y multicolinealidad. Para esto, la prueba Shapiro-Wilk de normalidad de los residuales entregó significancia marginal $(W=0.99, p=.047)$, pero una confirmación con la prueba Anderson-Darling sugiere normalidad $(A=0.65, p=.08)$; el test de Durbin-Watson indicó ausencia de autorrelación (valores críticos $0.06-1.86, p=.1$ ), mientras que el factor de inflación de varianza (VIF) con valores entre 1.01 (migración) y 2.21 (estrés) informó de poca probabilidad de multicolinealidad entre predictores; $y$, en las regresiones, las variables independientes se incluyeron en un solo bloque y fueron seleccionadas con argumentos principalmente teóricos. Todos los análisis fueron realizados con IBM-SPSS versión 24 (IBM Corp., 2016), y valores de probabilidad menores al $5 \%$ se consideraron como significativos.

\section{Resultados}

A continuación se describen los antecedentes médicos y la sintomatología de depresión y estrés de la cohorte al inicio de sus estudios universitarios, y se muestra la exploración de los factores relacionados con la percepción general de la salud y la calidad de vida, en congruencia con los objetivos del estudio.

Antecedentes médicos y sintomas de depresión y estrés

La Tabla 1 resume indicadores médicos y de salud emocional de la cohorte total y por sexo. En general, se aprecia una prevalencia relativamente baja (rango del 11 al $16 \%$ ) de enfermedad diagnosticada, limitación funcional, sobrepeso u obesidad y de estudiantes que requieren seguimiento médico; que en los estudiantes con diagnóstico de enfermedad (12.1\%), la enfermedad física es tres veces mayor (74\%) en comparación con la mental; que los síntomas de depresión y estrés por encima del nivel normal están presentes en el 27.2 y $30.7 \%$ respectivamente; y, por sexo, que en mujeres hay un porcentaje significativamente mayor de enfermedad diagnosticada $(p=.002)$, necesidad de seguimiento médico $(p=.01)$ y estrés por encima del nivel normal $(p=.04)$, así como menor sobrepeso u obesidad $(p=.002)$ según el IMC (véase Tabla 1$)$.

Tabla 1.

Antecedentes médicos, depresión y estrés

\begin{tabular}{|c|c|c|c|c|}
\hline & $\begin{array}{c}\text { Total (636) } \\
\mathrm{n}(\%) \\
\end{array}$ & $\begin{array}{c}\text { Mujeres (364) } \\
\mathrm{n}(\%)\end{array}$ & $\begin{array}{c}\text { Hombres (272) } \\
\mathrm{n}(\%)\end{array}$ & Valor $p$ \\
\hline Enfermedad diagnosticada ${ }^{a}$ & $77(12.1)$ & $57(15.7)$ & $20(7.7)$ & .002 \\
\hline Enfermedad física & $57(74)$ & $41(71.9)$ & $16(80)$ & \multirow{2}{*}{.47} \\
\hline Enfermedad mental & $20(26)$ & $16(28.1)$ & $4(20)$ & \\
\hline Discapacidad o limitación ${ }^{b}$ & $70(11)$ & $47(12.9)$ & $23(8.5)$ & .07 \\
\hline Seguimiento médico & $100(15.7)$ & 69(19) & $31(11.4)$ & .01 \\
\hline Sobrepeso/obesidad $^{c}$ & $85(13.8)$ & $35(10)$ & $50(18.9)$ & .002 \\
\hline Depresión ${ }^{\mathrm{d}}$ & $173(27.2)$ & $95(26.2)$ & $78(28.9)$ & .44 \\
\hline Estrés ${ }^{\mathrm{d}}$ & $195(30.7)$ & $123(34.9)$ & $72(27.3)$ & .04 \\
\hline
\end{tabular}

Nota. ${ }^{a}$ Reportada por el estudiante. ${ }^{\mathrm{b}}$ Para el desarrollo de actividades diarias. ${ }^{\mathrm{c}}$ IMC $>25 .{ }^{\mathrm{d}}$ Nivel leve a extremadamente severo. 
Factores relacionados con la percepción general de la salud y la calidad de vida

La Tabla 2 muestra la percepción general de la salud de buena a excelente y la calidad de vida en sus dimensiones física, psicológica, social y ambiental para la cohorte total y por factores sociodemográficos, médicos y de salud emocional. En general, se observa que casi la totalidad de los estudiantes percibe su salud de buena o excelente (91\%), indicador que es significativamente inferior en quienes tienen enfermedad diagnosticada $(80.5 \%)$ y síntomas de depresión $(86.1 \%)$ y estrés $(83.1 \%)$. En cuanto a la calidad de vida, la dimensión con menor puntaje promedio corresponde a la calidad de vida psicológica $(M=66.5)$, y la de mayor puntuación es para la dimensión ambiental $(M=71.6)$. Como información complementaria, la tabla muestra puntos de corte para tres grupos iguales (percentil 33 y 66), que pueden ser usados para crear categorías de calidad de vida (baja, media y alta) en poblaciones similares.
Al comparar por factores demográficos, se aprecia en mujeres menor calidad de vida física (diferencia de medias $=10.4)$, psicológica (6.3) y ambiental (4.1), y en migrantes menor calidad de vida física (3.5), social (5.1) y ambiental (4.8). Además, en estudiantes de nivel socioeconómico bajo, y entre quienes se financian con créditos o becas, se encontró menor calidad de vida ambiental (7.8 y 4.6 respectivamente), siendo significativas todas las diferencias (véase Tabla 2). Asimismo, en las variables médicas se observa menor calidad de vida física (6.4), psicológica (9) y ambiental (3.5) para estudiantes con reporte de enfermedad, y únicamente peor calidad de vida psicológica en el grupo con sobrepeso u obesidad (5.1). Finalmente, al comparar los grupos de estudiantes con y sin síntomas de depresión y estrés, se aprecia en ambos casos menor calidad de vida en todas dimensiones, siendo especialmente notables las diferencias para la calidad de vida psicológica tanto para la depresión (20.3) como para el estrés (16.6).

Tabla 2.

Factores de la percepción de salud y de la calidad de vida

\begin{tabular}{|c|c|c|c|c|c|}
\hline & \multirow{2}{*}{$\begin{array}{l}\text { Percepción global } \\
\text { de la salud } \\
(\%)\end{array}$} & \multicolumn{4}{|c|}{ Calidad de vida (WHOQOL-BREFF) } \\
\hline & & $\begin{array}{c}\text { Dominio físico } \\
M(D E)\end{array}$ & $\begin{array}{c}\text { Dominio psicológico } \\
M(D E)\end{array}$ & $\begin{array}{c}\text { Dominio social } \\
M(D E)\end{array}$ & $\begin{array}{c}\text { Dominio ambiental } \\
M(D E)\end{array}$ \\
\hline Todos (636) & & $69.1(13.9)$ & $66.5(17.4)$ & $67.3(20.6)$ & $71.6(14.1)$ \\
\hline Percentil 33 & 91 & 64.2 & $\mathrm{P} 33=62.5$ & $\mathrm{P} 33=58.3$ & $\mathrm{P} 33=64.2$ \\
\hline Percentil 66 & & 75 & P66 $=75$ & P66 $=75$ & $\mathrm{P} 66=78.1$ \\
\hline \multicolumn{6}{|l|}{ Sexo } \\
\hline Mujer (364) & 89 & $66.8 *(13.7)$ & 63.8*(17.3) & 66.1(19.9) & $69.9 *(14.1)$ \\
\hline Hombre (272) & 93 & $77.2(13.5)$ & $70.1(16.9)$ & $68.9(21.3)$ & $74(13.8)$ \\
\hline \multicolumn{6}{|l|}{ Estrato } \\
\hline Bajo (46) & 91.3 & $67.7(14.6)$ & $67(19)$ & $67(24.6)$ & $64.4 *(14.9)$ \\
\hline Medio/alto (576) & 91 & $69.2(13.8)$ & $66.5(17.3)$ & $67.4(20.2)$ & $72.2(13.7)$ \\
\hline \multicolumn{6}{|l|}{ Financiación } \\
\hline Créditos/becas (163) & 92 & $67.9(14)$ & $65.7(18.1)$ & $66.4(22)$ & $\mathbf{6 8 . 3} *(14.7)$ \\
\hline Familia (473) & 90.9 & $69.6(13.8)$ & $66.8(17.2)$ & $67.6(20.1)$ & $72.9(13.6)$ \\
\hline \multicolumn{6}{|l|}{ Migración } \\
\hline Sí (140) & 87.1 & $66.4 *(69.9)$ & $64.2(18)$ & $\mathbf{6 3 . 3} *(20.7)$ & $67.8 *(14)$ \\
\hline No (496) & 92.1 & $69.9(13.7)$ & $67.1(17.2)$ & $68.4(20.4)$ & $72.6(13.9)$ \\
\hline \multicolumn{6}{|l|}{ Enfermedad } \\
\hline Sí (77) & $80.5^{*}$ & 63.5*(12.4) & $\mathbf{5 8 . 6} *(19.3)$ & $64.2(20.5)$ & $68.6 *(14.3)$ \\
\hline No (559) & 92.6 & $69.9(13.9)$ & $67.6(16.9)$ & $67.7(20.5)$ & 72.1(14) \\
\hline \multicolumn{6}{|l|}{$I M C$} \\
\hline Sobrepeso/obesidad (85) & 88.2 & $67.3(14.4)$ & $\mathbf{6 2 . 3} *(17.6)$ & $65.7(20)$ & $71.2(13.1)$ \\
\hline Bajo/normal (529) & 91.5 & $69.6(13.8)$ & $67.4(17.3)$ & $68.1(20)$ & $72(14.1)$ \\
\hline \multicolumn{6}{|l|}{ Depresión } \\
\hline Leve o mayor (173) & 86.1* & $61 *(12.7)$ & $\mathbf{5 2 . 3 *} *(17.5)$ & $\mathbf{5 6 . 5} *(21.5)$ & $64.9 *(13.5)$ \\
\hline Normal (460) & 93.3 & $72.2(13)$ & $72(13.9)$ & $71.6(18.3)$ & $74.2(13.4)$ \\
\hline \multicolumn{6}{|l|}{ Estrés } \\
\hline Leve o mayor (195) & 83.1* & $62.4 *(12.4)$ & $\mathbf{5 5 . 3 *} *(17.5)$ & $\mathbf{5 8 . 3 *} *(21.9)$ & $\mathbf{6 5 *}(12.8)$ \\
\hline Normal (421) & 94.5 & $72.6(13.1)$ & $71.9(14.7)$ & $71.5(18.5)$ & $74.9(13.4)$ \\
\hline
\end{tabular}

Nota. $* p<.05$. En negrilla aparecen los resultados significativamente menores de salud percibida o calidad de vida. 
Predictores de calidad de vida física y psicológica

Con el objetivo de identificar predictores independientes - controlando el resto de las variables en el modelo - de calidad de vida en los dominios físico y psicológico, se incluyó el sexo, la migración por estudios, el nivel socioeconómico, la fuente de financiación de los estudios, el diagnóstico de enfermedad, el IMC, la depresión y el estrés, todos seleccionados por su posible aporte explicativo según la literatura especializada (véase Tabla 3 ).

Finalmente, en la Tabla 3 se evidencia una asociación significativa de la calidad de vida física con la depresión $(p<.001)$, el estrés $(p<.001)$, el sexo $(p<.001)$, la migración por estudios $(p<.001)$ y el IMC $(p<.001)$. Además, los coeficientes de regresión indican que menor estrés y depresión, sexo masculino, no migrar por estudios y menor IMC predicen calidad de vida en el dominio físico; no obstante, el alcance predictivo de estas cinco variables es limitado ( $26 \%$ de varianza explicada) y su reducción a solo cuatro, eliminando el IMC para obtener un modelo más parsimonioso, produce un cambio mínimo en el $\mathrm{R}^{2}$ ajustado (.24).

Con las mismas variables independientes, la regresión de la calidad de vida psicológica muestra una asociación significativa con la depresión $(p<.001)$, la enfermedad diagnosticada $(p<.02)$, el sexo $(p<.001)$, el estrés $(p<.002)$ $\mathrm{y}$ el IMC $(p<.001)$, todas en la dirección esperada. Los estudiantes de sexo masculino, con menor emocionalidad negativa - depresión y estrés-, sin enfermedad diagnosticada y menor IMC, presentan puntuaciones más altas de calidad de vida psicológica; modelo que resultó considerablemente mejor que el anterior (41\% de varianza explicada).

\section{Discusión}

El inicio de los estudios universitarios constituye un momento decisivo y complejo en la vida de los jóvenes, caracterizado por enormes expectativas y retos notables en la dimensión social y educativa. En ese sentido, los estudiantes de primer semestre enfrentan múltiples exigencias que a corto o mediano plazo pueden comprometer su proyecto educativo y también su salud (Azmitia et al., 2013; El Ansari et al., 2011).

Considerando los múltiples retos del primer semestre y los notables cambios en la composición social de las universidades en Colombia, en el presente trabajo se propuso identificar factores relacionados con la percepción de la salud y la calidad de vida en estudiantes de reciente ingreso a una universidad privada de tamaño grande (26 926 estudiantes de pregrado) en Colombia (Pontificia Universidad Javeriana, 2018). Esto con el fin de mejorar el conocimiento disponible y guiar las acciones de promoción de la salud, en especial desde una perspectiva positiva. Los hallazgos pueden ser usados para priorizar las necesidades de ciertos grupos de estudiantes.

En resumen, se encontró en la mayor parte de los estudiantes antecedentes médicos favorables tanto en hombres como en mujeres, una prevalencia baja de enfermedad diagnosticada (12.9\%), y una percepción dominante de salud como buena o excelente (91\%), panorama característico de la juventud en países de renta media y alta (Chu et al., 2015; El Ansari et al., 2011; Intra et al., 2011; Mikolajczy et al., 2008). La complementariedad entre las cifras anteriores sugiere que el reporte de salud buena o excelente puede estar esencialmente determinado por la ausencia de enfermedad, ya que son los estudiantes con diagnóstico de enfermedad física o mental, entre todos los segmentos analizados, quienes presentan menor percepción de salud buena o excelente $(80.5 \%)$. Sin embargo, una mejor comprensión de este particular demanda una aproximación cualitativa que indague el significado atribuido a la salud por parte de esta población.

Centrando la atención en la identificación de factores de calidad de vida, se llevaron a cabo dos aproximaciones:

Tabla 3.

Predictores independientes de calidad de vida física y psicológica

\begin{tabular}{|c|c|c|c|c|c|c|c|c|}
\hline \multirow{2}{*}{ Predictores } & \multicolumn{3}{|c|}{ Calidad de vida física $^{\mathrm{a}}$} & \multicolumn{5}{|c|}{ Calidad de vida psicológica $^{\mathrm{b}}$} \\
\hline & $\mathrm{B}$ & ES & $\beta$ & Valor $p$ & $\mathrm{~B}$ & ES & $\beta$ & Valor $p$ \\
\hline Sexo $($ Mujer $=1)$ & -4.46 & 1.03 & -0.16 & $<.001$ & -6.06 & 1.16 & -0.17 & $<.001$ \\
\hline Nivel socioeconómico $($ Bajo $=1)$ & -0.32 & 1.97 & -0.006 & .87 & 2.56 & 2.23 & -0.03 & .25 \\
\hline Migración $\left(\mathrm{S}_{1}=1\right)$ & -3.55 & 1.25 & -0.1 & .005 & -1.97 & 1.41 & -0.04 & .16 \\
\hline Financiación ${ }^{\mathrm{c}}($ Créditos/becas $=1)$ & 0.54 & 1.18 & 0.01 & .64 & 0.62 & 1.33 & 0.01 & .64 \\
\hline Enfermedad $(\mathrm{Sí}=1)$ & -2.64 & 1.55 & -0.06 & .08 & -3.85 & 1.75 & -2.2 & .02 \\
\hline $\mathrm{IMC}^{\mathrm{d}}$ & -0.33 & 0.16 & -0.07 & .03 & -0.42 & 0.18 & -0.07 & .02 \\
\hline Depresión $^{\mathrm{e}}$ & -0.39 & 0.08 & -0.24 & $<.001$ & -0.93 & -0.09 & -0.47 & $<.001$ \\
\hline Estrés ${ }^{\mathrm{e}}$ & -0.36 & 0.08 & -0.23 & $<.001$ & -0.29 & 0.09 & -0.14 & .002 \\
\hline
\end{tabular}

Nota. ${ }^{\text {a }}$ Dominio físico del WHOQOL-BREF $\left(R^{2}=0.26\right) .{ }^{\mathrm{b}}$ Dominio psicológico del WHQOL-BREF $\left(R^{2}=0.41\right) .{ }^{\mathrm{c}}$ Categoría de referencia $=$ recursos familiares. ${ }^{\mathrm{d}}$ Índice de masa corporal $\left(\right.$ peso/estatura $\left.{ }^{2}\right) .{ }^{\mathrm{e}}$ Puntuación continua del DASS-21 multiplicada por 2. 
66

la primera con una serie de análisis bivariados de variables sociodemográficas, médicas y de salud emocional — síntomas de depresión y estrés - reportadas por los estudiantes; y la segunda con un análisis multivariado orientado a controlar posibles variables de confusión (confounding), como, por ejemplo, la posible mayor presencia de mujeres dentro del grupo con migración por estudios.

Con la primera aproximación encontramos una calidad de vida inferior en las cuatro dimensiones en estudiantes con síntomas - por encima de lo normal— de depresión y estrés. En la misma línea, se encontró una calidad de vida inferior - en tres de cuatro dimensiones - en mujeres, en migrantes y en estudiantes con reporte de enfermedad física o mental. Adicionalmente, un efecto limitado a la dimensión ambiental en estudiantes de nivel socioeconómico bajo - 1 y 2 en Colombia en estratificación de viviendas - y que se financian con créditos o becas, así como una calidad de vida psicológica inferior en los estudiantes con sobrepeso $\mathrm{u}$ obesidad. Lo anterior confirma que en esta población las diferencias entre mujeres y hombres no se limitan al dominio psicológico, como se ha sido sugerido (Mikolajczy et al., 2008), pues también se aprecia inequidad a favor de los hombres en la calidad de vida física y ambiental.

Adicionalmente, este trabajo aporta evidencia de las necesidades especiales de estudiantes migrantes (Lolokote et al., 2017), fenómeno que es cada vez más común en esta población, por tratarse de ciudades receptoras ubicadas en el centro y sur occidente de Colombia (Bogotá y Cali). Con respecto a esta población, es importante notar que en otros estudios se ha encontrado peor evaluación de la salud general en estudiantes que viven solos o sin sus padres, situaciones seguramente secundarias a la migración por estudios (Chau \& Vilela, 2017; Mikolajczy et al., 2008).

Con respecto al hallazgo relativo al sobrepeso, la importancia de la imagen corporal en la adolescencia y su relación con las emociones (Lolokote et al., 2017) podrían explicar su menor bienestar psicológico. Particularmente para la cohorte de este proyecto, esta condición de riesgo médico es la única con menor prevalencia en mujeres ( $10 \%$ vs. $18.9 \%$ de hombres), como ha ocurrido en otras investigaciones (Mikolajczyk, 2008). Sin embargo, desconocemos si este menor sobrepeso/obesidad se mantendrá en el tiempo, pues la evidencia señala una mayor reducción de la actividad física en mujeres en la transición a la universidad (Han et al., 2008), fenómeno que no ha sido suficientemente estudiado en Colombia.

Finalmente, dentro de los análisis multivariables se detectó que la sintomatología emocional —depresión y estrés - presenta un mayor poder predictivo de la calidad de vida en la dimensión física y psicológica. Controlando las demás variables, la depresión, el estrés, ser mujer y la migración por estudios predicen inferior calidad de vida física; mientras que, por su parte, la depresión, tener una enfermedad, ser mujer, el estrés y el IMC alto predicen inferior calidad de vida psicológica. Este hallazgo extiende a universitarios colombianos de primer semestre la importancia atribuida a los factores psicológicos (Chau \& Saravia, 2014; Chu et al., 2015; Mikolajczy et al., 2008), sociales y físicos (Chau \& Saravia, 2014; Chu et al., 2015; Lolokote et al., 2017; Mikolajczy et al., 2008) en cuanto a la calidad de vida en sus diferentes dimensiones.

\section{Limitaciones del estudio}

Una limitación importante de este trabajo es su orientación exclusiva al uso de medidas de autorreporte. En lo posible, en estudios similares se debería hacer uso de otras fuentes de información, en especial para establecer los antecedentes médicos de los estudiantes. En este mismo aspecto, destaca en el trabajo de campo la dificultad de algunos para reportar su peso y estatura, medidas que son relativamente fáciles de obtener de forma objetiva. Otro asunto que amerita mención es la ausencia dentro de los análisis de comportamientos saludables y no saludables, los cuales podrían ser determinantes importantes tanto de la percepción de la salud como de la calidad de vida.

\section{Implicaciones para los programas de bienestar}

Por último, los hallazgos del estudio permiten a las universidades con población similar orientar sus programas de bienestar a la atención de grupos prioritarios, aprovechando la información sociodemográfica y de antecedentes médicos que regularmente se obtiene dentro de los procesos de ingreso. De este trabajo, además, surge la conveniencia de implementar procesos periódicos de tamizaje de la salud emocional de los nuevos estudiantes, considerando su estrecho vínculo con la percepción de la salud y con la calidad de vida. En este sentido, los jóvenes con diagnóstico de enfermedad, los migrantes y los estudiantes con niveles relevantes de depresión y estrés deben ocupar especial lugar dentro de los servicios y estrategias de promoción de la salud; esto sin perder de vista la situación diferencial de las mujeres que, según nuestra evidencia, va más allá de la dimensión psicológica.

\section{Referencias}

Ari, L. L., \& Shulman, S. (2012). Pathways of sleep, affect, and stress constellations during the first year of college: trasition difficulties of emergening adults. Journal of Youth Studies, 15(3), 273-292. https://doi.org/10.1080/13676261.2011.63 5196 
Arroyo, M., Rocandio , A. M., Ansotegui, L., Pascual, E., Salces, I., \& Rebato, E. (2006). Calidad de la dieta, sobrepeso y obesidad en estudiantes universitarios. Nutrición Hospitalaria, 21(6), 673-679. https://addi.ehu.es/bitstream/ handle/10810/13917/Nutr\%20Hosp\%202006_21_673-79. pdf? sequence $=1 \&$ isAllowed $=\mathrm{y}$

Ato, M., López, J., \& Benavente, A. (2013). Un sistema de clasificación de los diseños de investigación en psicología. Anales de Psicología, 29(3), 1038-1059. http://doi. org/10.6018/analesps.29.3.178511

Azmitia, M., Syed, M., \& Radmacher, K. (2013). Finding your Niche: Identity and Emotional Support in Emerging Adults' Adjusment to the Transition to College. Journal of Research on Adolescence, 23(4), 744-761. http://doi.org/10.1111/ jora. 12037

Barnett, N. P., Ott, M. Q., Rogers, M. L., Loxley, M., Linkletter, C., \& Clark, M. A. (2014). Peer associations for sustance use and exercise in a college student social network. Health Psychology, 33(10), 1134-1142. http://doi.org/10.1037/ a0034687

Bayram, N., \& Bilgel, N. (2008). The prevalence and sociodemographic correlations of depression, anxiety and stress among a group of university students. Social Psychiatry and Psychiatric Epidemiology, 43(8), 667-672. http://doi. org/10.1007/s00127-008-0345-x

Chau, C., \& Saravia, J. C. (2014). Adaptación universitaria y su relación con la salud percibida en una muestra de jóvenes de Perú. Revista Colombiana de Psicología, 23(2), 269-284. http://doi.org/ 10.15446/rcp.v23n2.41106

Chau, C., \& Vilela, P. (2017). Variables asociadas a la salud física y mental percibida en estudiantes universitarios de Lima. Liberabit, 23(1), 89-102. http://doi.org/10.24265/li berabit.2017.v23n1.06

Chu, J. J., Khan, M. H., Jahn, H. J., \& Kraemer, A. (2015). Comparison of Subjective Health Complaints between Chinese and German university Students: A cross-Sectional Study. International Journal of Environmental Research and Public Health, 12(12), 15794-15806. http://doi. org/10.3390/ijerph121215019

Conley, C. S., Durlak, J. A., \& Dickson, D. (2013). An evaluative review of outcome research on universal mental health promotion and prevention programs for higher education students. Journal of American College Health, 61(5), 228301. http://doi.org/10.1080/07448481.2013.802237

Conley, C., Traver, L. V., \& Bryant, F. B. (2013). Promoting psychosocial adjustment and stress management in firstyear college students: the benefits of engagement in a psychosocial wellness seminar. Journal of American College Health, 61(2), 75-86. http://doi.org/10.1080/07448 481.2012.754757

Dvoráková, K. K., Kishida, M., Li, J., Elawvsky, S., Broderick, P. C., Agrusti, M. R., \& Greenberg, M. T. (2017). Promoting healthy transition to college through mindfulness training with first-year college students: pilot randomized controlled trial. Journal of American College Health, 65(4), 259-267. http://doi.org/10.1080/07448481.2017.1278605

El Ansari, W., Stock, C., Snelgrove, S., Hu, X., Parke, S., Davies, S., John, J., Adetunji, H., Stoate, M., Deeny, P., Phillips, C., \& Mabhala, A. (2011). Feeling Healthy? A Survey of Physical and Psychological Wellbeing of Students from Seven Universities in the UK. International Journal of Environmental Reseach and Public Health, 8(5), 1308-1323. http://doi.org/10.3390/ijerph8051308

Fielder, R. L., Walsh, J. L., Carey, K. B., \& Carey, M. P. (2014). Sexual hookups and adverse health outcomes: a longitudinal study of first-year college women. Journal os Sex Research, 51(2), 131-144. http://doi.org/10.1080/0022449 9.2013.848255

Figuera, P., Dorio, I., \& Forner , A. (2003). Las competencias académicas previas y el apoyo familiar en la transición a la universidad. Revista de Investigación Educativa, 21(2), 349-369. https://revistas.um.es/rie/article/view/99251

Gravetter , F. J., \& Wallnau, L. B. (2013). Statistics for the Behavioral Sciences. Cengage Learning.

Han, J. L., Dinger, M. K., Hull, H. R., Randall, N. B., Heesch, K. C., \& Fields, D. A. (2008). Changes in women's physical activity during the transition to college. American Journal of Health Education, 39(4), 194-199. http://doi.org/10.1080 /19325037.2008.10599038

Hee-Kyung, J., Hyun-Ji, K., Young-Oh, K., Jae-Young, L., BeLong, C., Chun Soo, L., \& Sung-Eun, J. (2017). Health promotion in young adults at a university in Korea A crosssectional study of 625 participants in a university. Medicine, 96(7). https://doi.org/10.1097/MD.0000000000006157

IBM Corp. (2016). IBM SPSS Statistics for Windows, Version 24.0. IBM Corp.

Intra, M. V., Roales-Nieto, J. G., \& Moreno, E. (2011). Cambio en las conductas de riesgo y salud en estudiantes universitarios argentinos a lo largo del periodo educativo. International Journal of Psychology and Psychological Therapy, 11(1), 139-147. https://www.ijpsy.com/volumen11/num1/289/ cambio-en-las-conductas-de-riesgo-y-salud-ES.pdf

Lolokote, S., Habtemariam, T., \& Li, X. (2017). Do sociocultural factors influence students' self-rated health status and health-promoting lifestyles? A cross-sectional multicenter study in Dalian, China. BMC Public Health, 17(478). http://doi.org/10.1186/s12889-017-4411-8

Londoño, C. (2009). Optimismo y salud positiva como predictores de la adaptación a la vida universitaria. Acta Colombiana de Psicología, 12(1), 95-107. https:/actacolombianapsicologia.ucatolica.edu.co/article/view/335

Lovibond, S. H., \& Lovibond, P. F. (1995). Manual for the Depression Anxiety \& Stress Scales (2. ${ }^{\text {a }}$ ed.). Psychology Foundation.

Lumley, T., Diehr. P., Emerson, S., \& Chen, L. (2002). The importance of the normality assumption in large public health data sets. Anual Review of Public Health, 23, 151-169. https:// doi.org/10.1146/annurev.publhealth.23.100901.140546 
Maruish, M. E. (2011). User's manual for the SF-36v2 Health Survey (3. ${ }^{\mathrm{a}}$ ed.). QualityMetric.

McGrath, R. E. (2012). Quantitative Models in Psychology. American Psychological Association.

Mikolajczyk, R. T., Brzoska, P., Maier, C., Ottova, V., Meier, S., Dudziak, U., Ilieva, S., \& El Ansari, W. (2008). Factors associated with self-rated health status in university students: a cross-sectional study in three Europeans countries. BMC Public Health, 8(215). http://doi. org/10.1186/1471-2458-8-215

Ministerio de Educación Nacional [MEN]. (2015). ¿Qué porcentaje de los jovenes ingresa de manera inmediata a la educación superior? Ministerio de Educación Nacional. http://cms.mineducacion.gov.co/static/cache/binaries/arti cles-350451_recurso_11.pdf?binary_rand $=2306$

Patel, V., Flisher, A. J., Hetrick, S., \& McGorry, P. (2007). Mental health of young people: a global public-health challenge. Lancet, 9569(369), 1302-1313. http://doi. org/10.1016/S01406736(07)60368-7

Patrick, M. E., \& Lee, C. M. (2010). Sexual motivations and engagement in sexual behavior during the transition to college. Archives of Sexual Behavior, 39, 674-681. http://doi. org/10.1007/s10508-008-9435-9

Patton, G. C., Coffey , C., \& Sawyer, S. M. (2009). Global patterns of mortality in young people: a systematic analysis of population health data. Lancet, 9693(374), 881-892. http:// doi.org/10.1016/S0140-6736(09)60741-8

Patton, G. C., Coffey, C., Romaniuk, H., Mackinnon, A., Carlin, J. B., Degenhardt, L., \& Moran, P. (2014). The prognosis of common mental disorders in adolescents: a 14-year prospective cohort study. Lancet, 9926(383), 1404-1411. http:// doi.org/10.1016/S0140-6736(13)62116-9

Patton, G. C., Sawyer, S. M., Santelli, J. S., Ross, D. A., Afifi, R., Allen, N. B., Arora, M., Azzopardi, P., Baldwin, W., Bonell, C., Kakuma, R., Kennedy, E., Mahon, J., McGovern, T., Mokdad, A. H., Patel, V., Petroni, S., Reavley, N., Taiwo, K., Viner, R. M. (2016). Our future: a Lancet commission on adolescent health and wellbeing. Lancet, 10036(387), 2423-2478. http://doi.org/10.1016/S0140-6736(16)00579-1
Pontificia Universidad Javeriana. (2018). Javeriana en cifras 2017. http://www.javeriana.edu.co/documents/15838/9656 486/PUJEC17+V7.pdf/cc0f75fd-ff41-4f4c-aed7-0a3b1 888ce 14

Racette, S. B., Inman, C. L., Clark, R., Royer, N. K., Steger-May, K., \& Deusinger, S. S. (2014). Excercise and cardiometabolic risk factors in graduate students: a longitudinal, observational study. Journal of American College Health, 62(1), 47-56. http://doi.org/10.1080/07448481.2013.843535

Resnick, M. D., Catalano, R. F., Sawyer, S. M., Viner, R., \& Patton, G. C. (2012). Seizing the opportunities of adolescent health. Lancet, 379(9826), 1564-1567. http://doi. org/10.1016/S0140-6736(12)60472-3

Resolución 175 de 2018. Por medio de la cual se establece el Reglamento Operativo para el programa de créditos condonables Ser Pilo Paga 4. Diario Oficial No. 50.495 de 2 de febrero de 2018. Ministerio de Educación Nacional. https:// normograma.info/men/docs/pdf/resolucion_mineduca cion_0175_2018.pdf

Ruiz, F. J., García Martín, M. B., Suárez Falcón, \& Odriozola González, P. (2017). The hierarchical factor structure of the spanish versión of depression, anxiety and stress scale -21 . International Journal of Psychology and Psychological Therapy, 17(1), 97-105. https://www.ijpsy.com/volumen17/ num1/460/the-hierarchical-factor-structure-of-the-EN.pdf

Sawyer, S. M., Afifi, R. A., Bearing, L. H., Blakemore, S. J., Dick, B., Ezeh, A. A., \& Patton, G. C. (2012). Adolescence: a foundation for future health. Lancet, 379(9826), 16301640. https://doi.org/10.1016/S0140-6736(12)60072-5

The World Health Organization Quality of Life Group. (1994). Development of the WHOQOL: Rationale and current status. International Journal of Mental Health, 23(3), 24-56. http://doi.org/10.1080/00207411.1994.11449286

Wengreen, H. J., \& Moncur, C. (2009). Change in diet, physical activity, and body weight among young-adults during the trasition from high school to college. Nutrition Journal, 8(32). http://doi.org/10.1186/1475-2891-8-32

World Health Organization [WHO]. (1998). WHOQOL User Manual. WHO. 